\title{
Between tide and wave marks: a unifying model of physical zonation on littoral shores
}

The effects of tides on littoral marine habitats are so ubiquitous that shorelines are commonly described as 'intertidal', whereas waves are considered a secondary factor that simply modifies the intertidal habitat. However mean significant wave height exceeds tidal range at many locations worldwide. Here we construct a simple sinusoidal model of coastal water level based on both tidal range and wave height. From the patterns of emergence and submergence predicted by the model, we derive four vertical shoreline benchmarks which bracket up to three novel, spatially distinct, and physically defined zones. The (1) emergent tidal zone is characterized by tidally driven emergence in air; the (2) wave zone is characterized by constant (not periodic) wave wash; and the (3) submergent tidal zone is characterized by tidally driven submergence. The decoupling of tidally driven emergence and submergence made possible by wave action is a critical prediction of the model. On wavedominated shores (wave height >> tidal range), all three zones are predicted to exist separately, but on tide-dominated shores (tidal range $>>$ wave height) the wave zone is absent and the emergent and submergent tidal zones overlap substantially, forming the traditional "intertidal zone". We conclude by incorporating time and space in the model to illustrate variability in the physical conditions and zonation on littoral shores. The wave:tide physical zonation model is a unifying framework that can facilitate our understanding of physical conditions on littoral shores whether tropical or temperate, marine or lentic. 
1 Christopher E. Bird ${ }^{1,2}$, Erik C. Franklin², Celia M. Smith³ and Robert J. Toonen²

2

3

$4{ }^{1}$ Texas A\&M University - Corpus Christi, Department of Life Sciences, 6300 Ocean Drive,

5 Corpus Christi, TX 78412-5802

$6 \quad{ }^{2}$ Hawaii Institute of Marine Biology, University of Hawaii, P.O. Box 1346, Kaneohe, HI 96744

$7{ }^{3}$ Department of Botany, University of Hawaii, 3190 Maile Way, Honolulu, HI 96822 
9 Littoral habitats, those lying between the low-tide line and the upper limit of aquatic

10 species on the shore, are among the most studied and well-known aquatic habitats. Much

11 attention has been devoted to the study of organisms on rocky shores - in particular their vertical

12 zonation, the upper and lower limits of species, and distribution along gradients of wave

13 exposure. Hypotheses addressing the causes of biotic zonation and community structure have

14 evolved from strictly physical to an inseparable combination of physical and biological factors,

15 including physiological tolerance and species interactions. Throughout the progression of

16 intertidal zonation research, the most widely accepted paradigm has remained that the predictable

17 pattern of tidal rise and fall is the "primary" mechanism affecting shoreline water levels and the

18 littoral habitat.

19 Stephenson and Stephenson proposed their "universal features of zonation between tide-

20 marks on rocky coasts" after conducting surveys of vertical biotic zonation on littoral shores

21 world-wide. While the model of Stephenson and Stephenson (1972) focuses on biotic zonation,

22 it is essentially a physical model based on the concept that there is a classic intertidal zone

23 (midlittoral zone $=$ balanoid zone), a transition zone between the intertidal zone and the terrestrial

24 biosphere (supralittoral fringe $=$ Littorina zone), and a transition zone between the intertidal zone

25 and subtidal biosphere (infralittoral fringe = laminarian zone). One of the benefits of focusing on

26 the biota, rather than elevation, was that these zones were not found predictably at the same

27 height above sea level either within or among geographic regions. "Secondary modifying

28 factors" of littoral climate, such as waves, were thought to enlarge and/or elevate the basic zones,

29 but not change their primary properties . More recent research continues to demonstrate that

30 biotic zones and species distribution limits do not consistently occur at the same shore levels,

31 even within shores. 
A fundamental advance in the understanding of biotic zonation on rocky shores was the

33 demonstration that species interactions also affected zonation patterns, where biotic factors

34 generally determine the lower limit of distribution and physical factors affect the upper limit of

35 distribution. A number of exceptions to this generalization have been demonstrated, many of

36 which highlight the effect of biotic interactions on the realized distribution of a species. These

37 examples demonstrate that biotic factors can also regulate the upper limits of a species'

38 distribution, but focus on the proximate factors affecting realized species distributions.

39 Ultimately, the inseparable interaction between physical and biotic factors define the realized

40 limits of species, and models of physical clines can contribute valuable information to elucidate

41 biotic processes. Indeed,

42 'rocky shores are the 'stage' upon which ecological 'dramas' are played out, and physical conditions both provide the 'ambiance' and help direct the 'plays'."

44 Consequently, a more predictive model of physical habitat zonation on littoral shores would be 45 very valuable.

Patterns of changing community structure and composition along wave exposure

47 gradients have not typically been viewed as an issue of zonation. Instead, shifts in community

48 structure along wave exposure gradients have traditionally been associated with the effects of

49 hydrodynamic force (physical stress and disturbance) on biotic interactions. This is an

50 oversimplification, however, because with increasing wave exposure factors other than

51 hydrodynamic force are at play on shores where wave heights approach or exceed the tidal range.

52 More recent studies demonstrate that waves also affect patterns of community composition along

53 wave exposure gradients by creating habitat with associated physiological stress and disturbance

54 that does not exist on more wave protected shores. In particular, GIS-based models of wave

55 exposure and quantitative metrics of effective fetch can explain a considerable proportion of

56 variance in species abundances among sites at some locations. Even so, the manner and 
mechanism by which waves interact with tides to create littoral habitat and the subsequent effects

58 on biological processes have yet to be fully explored.

59 Here we investigate the roles of tides and waves in driving the characteristics of the

60 physical habitat by deriving a sinusoidal model of coastal water level. We begin with a model of

61 coastal water level based solely on tidal range and wave height. We use the model to derive

62 relevant physically-defined shoreline benchmarks and partition the littoral zone into novel

63 habitats. In so doing, we develop a system for the generic physical quantification and

64 categorization of any shoreline across a range of spatial and temporal scales.

First, we model the coastal water level $(W)$ over time by summing sinusoidal models of water level due to tidal $\left(W_{T}\right)$ and wave action $\left(W_{W}\right)$ as follows:

\section{Model of coastal water level}

We construct our simple model of coastal water level using both tidal and wave signals.

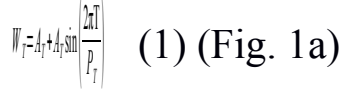

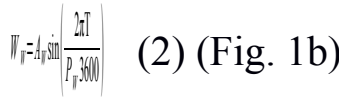

$$
\|=\|+\| \mathbb{T}(3)(\text { Fig. 1c) }
$$

74 where $A_{T}$ is the tidal amplitude in meters, $T$ is the time in number of hours, $P_{T}$ is the tidal period $75\left(P_{T}=12.2 \mathrm{~h}\right), A_{W}$ is the wave amplitude, and $P_{W}$ is the wave period $\left(P_{W}=10 \mathrm{~s}\right.$, note that the wave 76 period has been increased in the figures for aesthetic reasons). The goal of this model is to

77 grossly estimate the patterns of emersion and submersion experienced by shoreline organisms

78 and demonstrate the conceptual consequences of taking wave height into account. Many

79 additional factors interact with wave height to determine the extent of wave run-up and splash 
and will affect patterns of emersion and submersion, especially among regions with semi-diurnal,

81 diurnal and semi-mixed tidal regimes, but incorporating that complexity is beyond the scope of

82 this effort and does not change our conclusions or the implications of the concepts developed

83 herein.

\section{Wave height and tidal range data}

Global maps of tidal range and satellite data on significant wave height which will be exceeded $50 \%$ of the time were used to generate Fig. 3a. For Fig. 3b, significant wave heights from the KNMI/ERA-40 Wave Atlas and tidal range from the TOPEX/POSEIDON 6.2 (TPXO6.2) data sets were used. The KNMI/ERA-40 Wave Atlas data were derived from the reanalysis of oceanographic and atmospheric data with the European Centre of Medium-Range Weather Forecast's (ECMWF) Integrated Forecasting System coupled to the third generation wave forecast WAM model. The diurnal tidal range was computed from the average maximum daily range for each day (MHHW - MLLW) using 10 available tidal constituents from TPXO6.2 .

94 Wave height to tidal range ratios were calculated and mapped for a $2^{\circ} \times 2^{\circ}$ global grid (Fig. 3b) with Matlab 7.5 (MathWorks, Natick, Massachusetts) and ArcGIS 9.2 (ESRI, Redlands, California). Tide and wave heights can vary on small spatial scales; therefore, we expect there to be heterogeneity in the ratio of wave height to tidal range at smaller spatial scales than can be represented in a global map. For example, although the Hawaiian Archipelago is classified as wave-dominated, shorelines behind shallow reef crests which cause waves to break will be mostly tide-dominated. Microsoft Excel 2003 and Visual Basic for Applications (Microsoft

101 Corp., Seattle, Washington), was the software modeling environment used to generate all

102 statistics and figures unless otherwise noted above.

103 On a finer scale, we selected three specific sites that, on average, exhibit wave-dominated 104 (Mokapu, Hawai’i), co-dominated (Humboldt, California), and tide-dominated conditions 
105 (Portland, Maine) to illustrate how their differences affect model predictions (locations marked

106 with stars, Fig. 3a). Real, not predicted, historical data on significant wave height and tidal range

107 (MHHW - MLLW) were extracted from the National Oceanographic and Atmospheric

108 Administration (NOAA) National Data Buoy Center (www.ndbc.noaa.gov) and NOAA National

109 Ocean Service oceanographic products (www.tidesandcurrents.noaa.gov), respectively. The

110 wave buoys were located at Mokapu, Hawai'i (station 51202, 2000-2005), Humboldt South Spit,

111 California (station 46212, 2004-2005), and Portland, Maine (station 44007, 1982-2001). The tide

112 stations were located at Moku O Lo`e, Hawai‘i (station 1612480, National Ocean Service

113 Waimanalo tide correction applied), North Spit, California (station 9418767), and Portland,

114 Maine (station 8418150).

115

116

117

RESULTS

118

Derivation of shoreline benchmarks and zones

119 We begin by exploring the sinusoidal water level model which incorporates both tidal

120 range and wave height for a single tidal cycle (Eq. 3, Fig. 1c). Four specific benchmarks

121 associated with submersion and emersion can be derived from the basic model, Benchmarks 1-4

122 (Fig. 2). Benchmark one $\left(B_{l}\right)$ is the height of the upper reach of the wave crests at high tide and

123 is defined as:

124

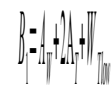

125 where $W_{\text {Tlow }}$ is the water level at low tide relative to MLLW (see Methods for definitions of other

126 variables). Benchmark two $\left(B_{2}\right)$ is the height of the upper reach of wave crests at low tide and is

127 defined as:

128

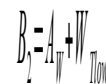


129 Benchmark three $\left(B_{3}\right)$ is the height of the lower reach of the wave troughs at high tide and is

130 defined as:

131

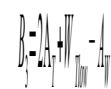

132 Benchmark four $\left(B_{4}\right)$ is the height of the lower reach of wave troughs at low tide and is defined 133 as:

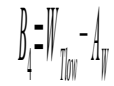

In the model, benchmarks one (blue) and four (black) mark the boundaries above which there is constant emersion and below which there is constant submersion, respectively (Fig. 2).

137 Benchmark two (green) marks the lowest position on the shore that experiences tidally induced

138 periods of emersion, and benchmark three (pink) marks the highest position on the shore that

139 experiences tidally induced submersion. Benchmarks 1-4 can be used to define discrete shoreline zones. Benchmarks one (blue) and two (green) bracket a zone of tidally induced emersion (Figs. $2 \mathrm{~h}, \mathrm{i})$, that we term the emergent tidal zone (vertical bars Figs. 2e,f). Similarly, benchmarks three

142 (pink) and four (black) demarcate a zone of tidally induced submersion (Figs. 2 h,i), that we label

143 the submergent tidal zone (horizontal bars, Figs. 2e,f). Where these zones overlap (Fig. 2d,g), a

144 zone of tidally induced emersion and submersion occurs - the traditional notion of an "intertidal

145 zone" and roughly equivalent to the mid littoral zone of Stephenson and Stephenson . A third

146 zone, bracketed by benchmarks two (green) and three (pink), is sandwiched between the

147 emergent and submergent tidal zones when wave height is greater than tidal range and is termed

148 the wave zone because it is washed by waves at both high and low tide (Fig. 2f,i).

\section{Derivation of physical categories for shorelines}

151 Our model of shoreline water level predicts three primary categories for intertidal shores:

152 tide-dominated, wave-dominated, and co-dominated (Fig. 2). When a shore is tide-dominated, 
153 tidal range is much greater than wave height and there is substantial overlap of the emergent and

154 submergent tidal zones (Figs. 2a,d,g). When a shore is wave-dominated, wave height is much

155 greater than tidal range and all three zones (emergent, wave, and submergent) exist independently

156 (Figs. 2c,f,i). The third category, co-domination, occurs when wave height = tidal range, and is

157 characterized by non-overlapping emergent and submergent intertidal zones, but no wave zone

158 (Figs. 2b,e,h).

159

160

\section{Global categorization of shorelines}

161

A strength of our intertidal zonation model is that it can be applied across a variety of

162 spatial and time scales. At a global spatial scale over a number of years, averaged data for wave

163 height and tidal range can be used to classify shores as tide-, wave-, or co-dominated (Fig. 3).

164 The most striking feature of Fig. 3 is that the majority of offshore oceanic islands are predicted to

165 be wave-dominated (Fig. 3b) and the majority of continental shores are predicted to be tide-

166 dominated on average (Fig. 3a). There are also numerous areas of co-domination and wave-

167 domination on continental shores. Wave-dominated shores are most prevalent in the Southern

168 Ocean where winds blow around the globe with few land barriers and in freshwater lakes which

169 have negligible tidal action. It should be noted that tidal range and wave heights can vary on

170 small spatial scales; therefore, we expect there to be heterogeneity in the ratio of wave height to

171 tidal range at smaller scales than can be represented in an averaged global map (see the section

172 below). This is not a failure of the model, rather it is an issue with the scale of the data loading

173 the model. For example, although the Hawaiian Archipelago is classified as wave-dominated,

174 shorelines behind shallow reef crests which cause waves to break will be mostly tide-dominated.

175 If wave height data were collected on a fine scale to decipher fore reef from back reef, this

176 pattern would be captured. Additionally, there is temporal variation in tidal range and wave 
177 heights which will cause temporal variation in the positions of Benchmarks 1-4, and could lead to

178 temporal variation in the classification of points on the shore as tide-, co-, or wave dominated.

\section{Temporal variation in zonation}

It is important to recognize that there is a continuum from tide-, to co-, to wave-

182 domination with no hard and fast boundaries, and the positions of Benchmarks 1-4 move as tidal

183 range and wave height varies. Although the long term averages presented in Figs. 3 and 4a-f are

184 informative, tidal range and wave heights are highly variable in time. Consequently, it is

185 important to be able to evaluate the variation in the position of Benchmarks 1-4 and the

186 associated physical littoral zones through time. We applied Equations 4-7, for predicting the

187 shore level of Benchmarks 1-4, to hourly wave height and tide measurements taken at Portland,

188 Maine; Humboldt, California; and Mokapu, Hawaii from June 2005 - June 2006 to evaluate the

189 variance in the predicted positions of the shoreline benchmarks on a fine temporal scale for one

190 year (Figs. $4 \mathrm{~g}$-i). The low tide line $\left(W_{\text {Tlow }}\right)$ and tidal amplitude $\left(A_{T}\right)$ for Equations $4-7$ were

191 defined as the low tide level and the tidal amplitude during each semidiurnal tidal period,

192 maximizing variation induced by tides.

193 As expected, there was considerable fine scale variation in the model-predicted positions

194 of the benchmarks at all three sites, which varied by up to $3.8 \mathrm{~m}, 4.4 \mathrm{~m}$, and $2.0 \mathrm{~m}$ at Portland,

195 Humboldt, and Mokapu, respectively (Figs. 4g-i). Portland is classified as tide-dominated (ratio

196 of wave height to tidal range $<0.5$ ) for $82 \%$ of the year-long period (Fig. $4 \mathrm{j}$, notice that $\mathrm{B}_{2}$ is

197 consistently lower on the shore than $\mathrm{B}_{3}$ indicating the overlap of the emergent intertidal and

198 submergent tidal). Mokapu is classified as wave-dominated (ratio of wave height to tidal range >

199 1.5) for $99 \%$ of the year-long period (Fig. 41, notice that $\mathrm{B}_{2}$ is consistently higher on the shore

200 than $\left.\mathrm{B}_{3}\right)$. 
Humboldt is an interesting case because it is predicted to be co-dominated for $48 \%$ and

202 wave-dominated for $48 \%$ of the year-long period on a fine temporal scale (Fig. 4k), but was

203 classified as co-dominated based on the yearly averaged diurnal tidal range and yearly mean

204 significant wave height (Fig. 4b). While there were portions of the winter where the significant

205 wave heights were consistently at least 1.5 times greater than the diurnal tidal range due to large

206 waves, most wave-dominated conditions were caused by large differences in the daily

207 semidiurnal tidal ranges - the smaller tidal range being wave-dominated and the greater tidal

208 range being co-dominated (causing the benchmark lines to appear thick in Fig. 4h). Ultimately,

209 Humboldt tends to be co-dominated by waves and tides on a diurnal scale in the summer and can

210 be wave-dominated in the winter, but also tends to alternate between co-domination and wave-

211 domination on a fine semidiurnal temporal scale. Overall, our model of coastal water level can

212 be used to effectively illustrate how patterns of wave and tide induced immersion vary at the

213 scale of a single tidal period and hundreds of tidal periods, facilitating the physical description of

214 sites in a concise and informative manner.

\section{DISCUSSION}

We sought to develop a model of coastal water level that incorporates tidal range as well as wave height to better understand patterns of immersion and emersion on shorelines. The wave-tide model of shoreline water level, composed of two sinusoidal signals (Fig. 1), is simple, highly scalable, and can be used to predict fairly complex patterns in coastal water level. The

221 model predicts the existence of four discrete shoreline benchmarks based upon patterns of 222 submersion and emersion (Fig. 2a-c). These four benchmarks bracket up to three primary

223 physical zones on littoral shores (Fig. 2f): (1) the zone that is continuously emergent at low tide

224 (emergent tidal zone), (2) the zone that is continuously submerged at high tide (submergent tidal 225 zone), and (3) the zone that is continuously washed by waves regardless of tidal level (wave 
226

227

228

229

230

231

232

233

234

235

236

237

238

239

240

241

242

243

244

245

246

247

248

249

250

zone). The magnitude of wave height relative to tidal range controls the existence of these zones, and can be used to categorize shores as tide-, co-, or wave-dominated (Fig. 2).

The most familiar condition is that of tide-domination, where a large portion of the shore

is characterized by tidally induced periods of both emersion and submersion - characteristics that

are accurately predicted by our model with an overlap of the emergent and submergent tidal

zones (Figs. 2a,d). For the most part, the world's shores have been perceived to be subject to

these tide-dominated conditions with only secondary modifications from wave action and other

factors (Ricketts et al. 1985). The wave-tide model, however, predicts large portions of the

earth's shores are co-dominated and even wave-dominated (Fig. 3), where tidally induced

emersion and submersion are spatially disconnected on the high and low shore, respectively (Fig.

2). Under co- and wave-dominated conditions, no position on the shore is predicted to

experience periods of both emersion and submersion on a tidal schedule, unlike the classic

“intertidal" conceptual model. Positions higher on the shore are alternately emerged and washed

by waves due to tidal action (emergent tidal zone) while the low shore is alternately submerged

and washed by waves (submergent tidal zone) (Fig. 2). During wave-dominated conditions, the

mid shore is perpetually washed by waves regardless of tide (wave zone), thus waves modulate

the vertical positions of emersion and submersion on shorelines. Importantly, because tidal range

is variable and wave height is highly variable, iteration of the model over time and space predicts

that the size and position of these zones will be highly variable (Fig. 4).

as well as fine temporal scales and midrange spatial scales, but it is also worthwhile to note that our shoreline zonation model can be applied at fine spatial scales of $\mathrm{cm}$ to $\mathrm{m}$. Waves and more importantly, wave run-up can be influenced by many factors such as bathymetry, shoreline 
251 topography, slope, aspect, porosity, and rugosity. The model's wave height parameter could be

252 replaced with wave run-up parameters and it will reflect differences on fine spatial scales.

253 Varying levels of wave exposure will affect the zonation predicted by the model. For example,

254 shallow reefs can cause waves to break, severely reducing wave height and run-up at the

255 shoreline, relative to that recorded at a wave buoy or coarse satellite imagery. Even within a

256 shore that has fairly consistent wave exposure, there are areas where waves run up higher than

257 others. If a shoreline were outfitted with submersion sensors, the positions of the benchmarks

258 and zones could be estimated. Indeed, fine scale analysis requires fine scale data.

\section{Behavior of zones with varying wave exposure and tidal range}

Increasing wave exposure is believed to simply elevate and expand the tidally defined

zones on the shore - a concept that has remained unchallenged in the literature. However, this

263 behavior is not predicted by the model we present. The position of the emergent tidal zone

264 (vertical bars, Figs. 2d-f) rises but that of the submergent tidal zone (horizontal bars, Figs. 2d-f)

265 sinks on the shore as wave height increases. The wave zone (wavy horizontal bars, Fig. 2f)

266 expands, but the emergent and submergent tidal zones remain the same size as wave height

267 increases. Therefore, increasing wave height while holding tidal range constant displaces the

268 tidal zones both higher and lower on the shore; this leads to the contraction of the region of

269 overlap between the two tidal zones, as well as the emergence and expansion of the wave zone

270 (see progression from Fig. 2d to Fig. 2f). Increasing tidal range, while holding wave height

271 constant, increases the size of emergent and submergent tidal zones which leads to the

272 contraction of the wave zone, and the expansion of the emergent-submergent overlap zone. Thus,

273 the effect of tidal range and wave height fluctuations over time on physical shore zones is

274 predicted to be different than previously envisioned. 


\section{Physical properties and biological implications of the wave:tide zonation model}

At a gross level, the model-predicted zones and their unique physical characteristics can

278 be used to further predict likely sources of stress and disturbance on biological communities.

279 (Fig. 2g-i, Table 1). The pattern and duration of immersion and emersion can have major effects

280 on the foraging of predators and physiological stresses such as temperature, desiccation, and

281 irradiation. As the physical zones move due to temporal variability in wave height and tidal

282 range, conditions are predicted to change, thereby influencing resident shoreline organisms.

283 Emergent tidal zone- The emergent tidal zone is characterized by tidally-influenced

284 periods of wave-wash and exposure to air (Fig 2). Therefore, the substratum temperature will be

285 alternately affected by the water, atmosphere and solar irradiation on a tidally defined cycle. The

286 substratum temperature amplitude can be much greater in this zone than the other littoral zones

287 we have defined. The level of drying can be great during low tides, depending on wave splash

288 and humidity, and the level of hydrodynamic force will oscillate from substantial to negligible on

289 a tidally defined cycle. Consequently, the biota residing in the emergent tidal zone will likely

290 contend with the physiological stresses of temperature and desiccation when exposed to the

291 atmosphere and the physical stress of wave force when wetted by the ocean. Terrestrial

292 predators, such as birds, will have access to the emergent tidal zone as the tide recedes, but non-

293 benthic aquatic predators, such as fish, will have limited access to this zone because it will be

294 constantly washed by waves at high water. Benthic marine predators will have to contend with

295 physiological stresses when exposed to the atmosphere and physical stresses when exposed to the

296 aquatic sphere, potentially limiting their efficiency .

297 Wave zone- Physiological stresses, such as temperature and desiccation, are minimal in

298 the wave zone because it is constantly washed by waves, and the temperature will be largely

299 affected by the ocean temperature, muting temperature amplitude relative to the emergent tidal

300 zone (Fig. 2). On the other hand, physical stress, in the form of hydrodynamic force caused by 
301 wave action, will be constant and the wave zone will receive more wave energy than the

302 emergent or submergent tidal zones. As a result of these conditions, terrestrial and aquatic

303 predators will have to contend with constant wave wash to safely access the wave zone. It is

304 likely that predation will be severely reduced in the wave zone relative to any other littoral zone .

305 Submergent tidal zone-Heat and desiccation stresses are negligible in the submergent

306 tidal zone because it is either washed by waves at low tide or completely submerged at high tide,

307 but never fully emergent (Fig. 2). Physical stress will be magnified during periods of wave wash.

308 Terrestrial predators will have little access to the submergent tidal zone, without specific

309 adaptations, but aquatic predators will have easy access during high tides.

$310 \quad$ Overlapping of the emergent and submergent tidal zones- These descriptions of the

311 emergent tidal, submergent tidal, and wave zones only apply to co- and wave-dominated

312 conditions. During tide-dominated conditions (Fig $2 \mathrm{a}, \mathrm{d}, \mathrm{g}$ ) the emergent and submergent tidal

313 zones overlap (overlapping vertical and horizontal bars, Fig. 2d). The result of the overlap

314 between the zones is that the area is characterized by discrete tidally defined periods of exposure

315 to the atmosphere, wave wash, and submergence - the conditions traditionally ascribed to a

316 classic "intertidal” zone (Fig 2. g). Biota residing in this area of overlap are subject to

317 physiological stresses (temperature, desiccation, and irradiation) during low water, are submerged

318 at high water, and are exposed to physical stress in the wave wash at some point between high

319 and low water. Terrestrial and aquatic predators have windows of foraging opportunity at low

320 and high water, respectively, but are restricted during the periodic wave wash events with the

321 tidal rise and fall .

322 The non-overlapping regions of the emergent tidal (vertical bars, Fig. 2d) and submergent

323 tidal zones (horizontal bars, Fig. 2d) share properties with those zones under co- and wave-

324 dominated conditions, but the periods of exposure to the atmosphere and submergence in the

325 emergent and submergent tidal zones, respectively, are long relative to the periods of wave wash. 
326 Thus, physical stress caused by waves will be reduced to shorter periods of time in both the

327 emergent and submergent tidal zones under tide-dominated conditions. Physiological stress is

328 potentially great and predation by swimming aquatic predators should be limited in the non-

329 overlapping portion of the emergent tidal zone. In contrast, physiological stress will be minimal

330 and access by aquatic predators will only be limited only during short periods of wave wash in

331 the non-overlapping portion of the submergent tidal zone.

\section{Consequences of variability in tidal range and wave height}

While the general qualitative conditions we attributed to the physical zones on littoral

335 shores are predicted to remain relatively constant over time, significant variation in both tidal

336 range and wave height is expected within sites as well as among them. This variability within

337 sites can lead to variation in the position and existence of these zones on a variety of time scales

338 (Figs. 2g-i). Against this backdrop of long-term average conditions, any given position on a

339 shore can be characterized by a temporary profile of another of the physical zones we have

340 described. Given that stresses and disturbances are often the result of uncommon events, rare

341 departures from average physical conditions are likely to be events of extreme stress and

342 disturbance. For example, $\sim 0.5 \mathrm{~m}$ above MLLW at Mokapu, HI is almost always predicted to be

343 in the wave zone (below $B_{2}$ and above $B_{3}$, Fig. 2i), but is occasionally in the lower emergent tidal

344 zone. On these rare occasions where $0.5 \mathrm{~m}$ is in the emergent tidal zone, physical stress due to

345 constant wave wash will be alleviated but physiological stress due to temperature, desiccation,

346 and solar radiation, as well as foraging by terrestrial predators are much more likely to impact the

347 biota.

348 In Portland, ME, $\sim 1.5 \mathrm{~m}$ above MLLW is predicted to be typically in the overlap of the

349 emergent and submergent tidal zones, but in approximately 23 discrete high-wave events it is

350 solely in the upper submergent tidal zone or the wave zone (Fig. 2g). During these rare events, 
351 physiological stress is expected to be greatly reduced, but physical stress is expected to be

352 elevated. Overall, both the average long-term conditions and the impact of rare events are

353 expected to be important determinants of community structure. Our model aids in the

354 identification of both the long term average conditions and these rare stress/disturbance events

355 and facilitates the generation of specific predictions of how each will affect resident biota at

356 specific positions on the shore.

357 When considering the additional complexities of the effects of bathymetry on wave height

358 and the effects of shore topography, slope, porosity, effective fetch, and rugosity on wave run-up,

359 it is easy to foresee that the model-predicted environmental conditions, based solely on coarse

360 scale measurements of wave height and tidal range, may not exactly match the actual conditions

361 at particular positions on the shore. The model is only as good as the data driving it, thus fine

362 scale tests of the model must include fine scale wave run-up and tidal data (e.g., Harley \&

363 Helmuth 2003; Burrows et al. 2008). The power of the model lies in its scalability and its ability

364 to identify transitions from tide- to co- to wave-domination, define typical conditions and

365 significant departures from the norm, and make general predictions about the stresses affecting

366 the biotic processes operating on any given shore.

\section{Relevance of a physical zonation model}

The use of physical zonation as a tool to better understand ecological processes on littoral

shores has been refuted by Underwood who demonstrated there are no sharp changes in

371 inundation patterns due to tidal ebb and flow, and thus, no zonation. Our model of shoreline

372 water level and physical zonation is predicated on the smooth sinusoidal behavior of tidal ebb and

373 flow, and is thus wholly consistent with the findings of Underwood (1978). Yet, we identify

374 critical, physically-defined benchmarks on the shore when considering wave action as well as

375 tidal fluctuation. We further agree with Underwood (1978) that particular species are not likely 
376 to be strictly limited within or by these zones. We propose, however, that the conditions

377 associated with these zones and their positions (as determined by the ratio of wave height to tidal

378 range) will have prominent effects on physical and physiological stress and disturbance

379 experienced by shoreline populations. The differential stresses in each zone will impact

380 prominent biotic interactions such as predation, competition, and facilitation . Consequently, our

381 model of physical zonation is an effective tool that can inform and facilitate our understanding of

382 physical processes operating on littoral shores, and is a fundamental advancement beyond the

383 classic intertidal concept.

\section{Conclusion}

Our model of coastal water level indicates that waves do not simply expand and elevate physical littoral zones that exist in the absence of waves. Rather, waves interact with tides to create up to three distinct physical zones (emergent tidal zone, wave zone, submergent tidal zone) that are characterized by unique submersion-emersion and hydrodynamic characteristics. The differential properties of these zones and the variability in their existence, overlap, and positions leads to specific and falsifiable hypotheses regarding differential regimes of physical and

392 physiological and stress and disturbance for organismal shoreline populations. What is more, our model of littoral zonation is completely consistent with the findings of previous studies that have argued against the existence of intertidal zonation (Underwood 1978, Benedetti-Cecchi \& Cinelli 1997), and is not hampered by a complete dependence on tidal patterns, geographic location, or the biological definition of zones. Thus, this model provides a unifying framework to better understand the physical littoral habitat and biotic stress regimes on shores, whether they are

398 temperate, tropical, marine, or lentic. 
400 Acknowledgements. We thank K. Bridges, D. Duffy, L. Freed, A. Kay, H. Zaleski, and

401 anonymous reviewers for providing insightful comments and constructive criticism. This is

402 HIMB contribution HIMB 1565, and SOEST 8988. 
404

405

406

407

408

409

410

411

412

413

414

415

416

417

418

419

420

421

422

423

424

425

426

427

428

429

430

431

432

433

434

435

436

437

438

439

440

441

442

443

444

445

446

447

448

449

450

Benedetti-Cecchi L, and Cinelli F. 1997. Spatial distribution of algae and invertebrates in the rocky intertidal zone of the Strait of Magellan: are patterns general? Polar Biology 18:337-343.

Bertness MD. 1989. Intraspecific competition and facilitation in a northern acorn barnacle population. Ecology 70:257-268.

Bertness MD, and Leonard GH. 1997. The role of positive interactions in communities: Lessons from intertidal habitats. Ecology 78:1976-1989.

Bertness MD, Leonard GH, Levine JM, Schmidt PR, and Ingraham AO. 1999. Testing the relative contribution of positive and negative interactions in rocky intertidal communities. Ecology 80:2711-2726.

Bruno JF, and Bertness MD. 2001. Habitat modification and facilitation in benthic marine communities. In: Bertness MD, Gaines SD, and Hay ME, eds. Marine community ecology. Sunderland, MA: Sinaur Associates, Inc., 201-218.

Burrows MT, Harvey R, and Robb L. 2008. Wave exposure indices from digital coastlines and the prediction of rocky shore community structure. Marine Ecology Progress Series 353:1.

Caires S, and Sterl A. 2005a. 100-year return value estimates for ocean wind speed and significant wave height from the ERA-40 data. Journal of Climate 18:1032-1048.

Caires S, and Sterl A. 2005b. A new non-parametric method to correct model data: Application to significant wave height from the ERA-40 reanalysis. Journal of Atmospheric and Oceanic Technology 22:443-459.

Choat JH. 1977. The influence of sessile organisms on the population biology of three species of acmaeid limpets. Journal of Experimental Marine Biology and Ecology 26:1-26.

Colman JS. 1933. The nature of intertidal zonation in plants and animals. J Mar Biol Assoc UK 18:435-476.

Connell JH. 1961a. Effects of competition, predation by Thais lapillus, and other factors on natural populations of the barnacle Balanus balanoides. Ecological Monographs 31:61104.

Connell JH. 1961b. The influence of interspecific competition and other factors on the distribution of the barnacle Chthamalus stellatus. Ecology 42:710-723.

Davies JL. 1980. Geographical variation in coastal development. London: Longman.

Denny MW, and Wethey DS. 2001. Physical processes that generate patterns in marine communities. In: Bertness MD, Gaines SD, and Hay ME, eds. Marine Community Ecology. Sunderland, MA: Sinauer Associates, Inc., 3-38.

Doty MS. 1946. Critical tidal factors that are correlated with the distribution of marine algae and other organisms along the Pacific coast. Ecology 27:315-328.

Egbert GD, Bennett AF, and Foreman MGG. 1994. TOPEX/POSEIDON tides estimated using a global inverse model. Journal of Geophysical Research 99:821-824.

Egbert GD, and Erofeeva SY. 2002. Efficient inverse modeling of barotropic ocean tides. Journal of Atmospheric and Oceanic Technology 19:183-204.

Garrity SD. 1984. Some adaptations of gastropods to physical stress on a tropical rocky shore. Ecology 65:559-574.

Garrity SD, Levings SC, and Caffey HM. 1986. Spatial and temporal variation in shell crushing by fishes on rocky shores of Pacific Panama. Journal of Experimental Marine Biology and Ecology 103:131-142.

Harley CDG. 2003. Abiotic stress and herbivory interact to set range limits across a twodimensional stress gradient. Ecology 84:1477-1488.

PeerJ reviewing PDF | (v2013:07:637:1:0:ACCEPTED 29 Aug 2013) 
Harley CDG, and Helmuth BST. 2003. Local- and regional-scale effects of wave exposure, thermal stress, and absolute versus effective shore level on patterns of intertidal zonation. Limnol Oceanogr 48:1498-1508.

Haslett SK. 2000. Coastal Systems. London: Routledge.

Hughes SA. 2004. Estimation of wave run-up on smooth, impermeable slopes using the wave momentum flux parameter. Coastal Engineering 51:1085-1104.

Janssen PAEM, Doyle JD, Bidlot J, Hansen B, Isaksen L, and Viterbo P. 2002. Impact and feedback of ocean waves on the atmosphere. In: Perrie WA, ed. Advances in Fluid Mechanics, Atmosphere-Ocean Interactions. Southampton, U.K.: WITpress, 155-197.

Komen GJ, Cavaleri L, Donelan M, Hasselmann K, Hasselmann S, and Janssen PAEM. 1994. Dynamics and Modelling of Ocean Waves. Cambridge, U.K.: Cambridge University Press.

Lewis JR. 1964. The ecology of rocky shores. London,: English Universities Press.

McLay CL, and McQueen DJ. 1995. Intertidal zonation of Cyclograpsus lavauxi H.Milne Edwards, 1853 (Brachyura:Grapsidae) along the coast of the South Island of New Zealand. Crustac Res:49-64.

Menge BA. 1978. Predation intensity in a rocky intertidal community. Relation between predator foraging activity and environment harshness. Oecologia 34:1-16.

Menge BA, and Branch GM. 2001. Rocky intertidal communities. In: Bertness MD, Gaines SD, and Hay ME, eds. Marine community ecology. Sunderland, MA: Sinauer Associates, Inc., 221-252.

Menge BA, and Olson AM. 1990. Role of scale and environmental factors in regulation of community structure. Trends in Ecology \& Evolution 5:52-57.

Menge BA, and Sutherland JP. 1976. Species diversity gradients: synthesis of the roles of predation, competition, and temporal heterogeneity. American Naturalist 110:351-369.

Menge BA, and Sutherland JP. 1987. Community regulation: variation in disturbance, competition, and predation in relation to environmental stress and recruitment. Amer Zool 130:730-757.

Paine RT. 1974. Intertidal community structure. Experimental studies in the relation between a dominant competitor and its principle predator. Oecologia 15:93-120.

Ricketts EF, Calvin J, Hedgpeth JW, and Phillips DW. 1985. Between Pacific Tides. Stanford, Calif:: Stanford University Press.

Robles C, and Desharnais R. 2002. History and current development of a paradigm of predation in rocky intertidal communities. Ecology 83:1521-1536.

Somero GN. 2002. Thermal physiology and vertical zonation of intertidal animals: Optima, limits, and costs of living. Integrative and Comparative Biology 42:780-789.

Stephenson TA, and Stephenson A. 1949. The universal features of zonation between tide-marks on rocky coasts. $J$ Ecol 37:289-305.

Stephenson TA, and Stephenson A. 1972. Life between tidemarks on rocky shores. San Francisco,: W. H. Freeman.

Sterl A, and Caires S. 2005. Climatology, Variability and Extrema of Ocean Waves - The Webbased KNMI/ERA-40 Wave Atlas. International Journal of Climatology 25:963-997.

Thomas ML. 1986. A physically derived exposure index for marine shorelines. Ophelia 25:1-13.

Underwood AJ. 1978. A refutation of critical tidal levels as determinants of the structure of intertidal communities on British shores. Journal of Experimental Marine Biology and Ecology 33:261-276.

Wootton JT. 1992. Indirect effects, prey susceptibility, and habitat selection: Impacts of birds on limpets and algae. Ecology 73:981-991. 
499 Young IR, and Holland G. 1996. Atlas of the oceans : wind and wave climate. Tarrytown, N.Y.:

$500 \quad$ Pergamon, Oxford

501

502

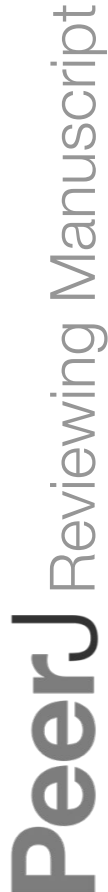




\section{Figure 1}

Model of shoreline water level over a single tidal cycle.

Representation of water level incorporating (a) only tidal range, (b) only wave height, and (c) both tidal range and wave height. The tidal amplitude $\left(A_{T}\right)$, tidal period $\left(P_{T}\right)$, wave amplitude $\left(A_{W}\right)$, and wave period $\left(P_{W}\right)$ are noted.

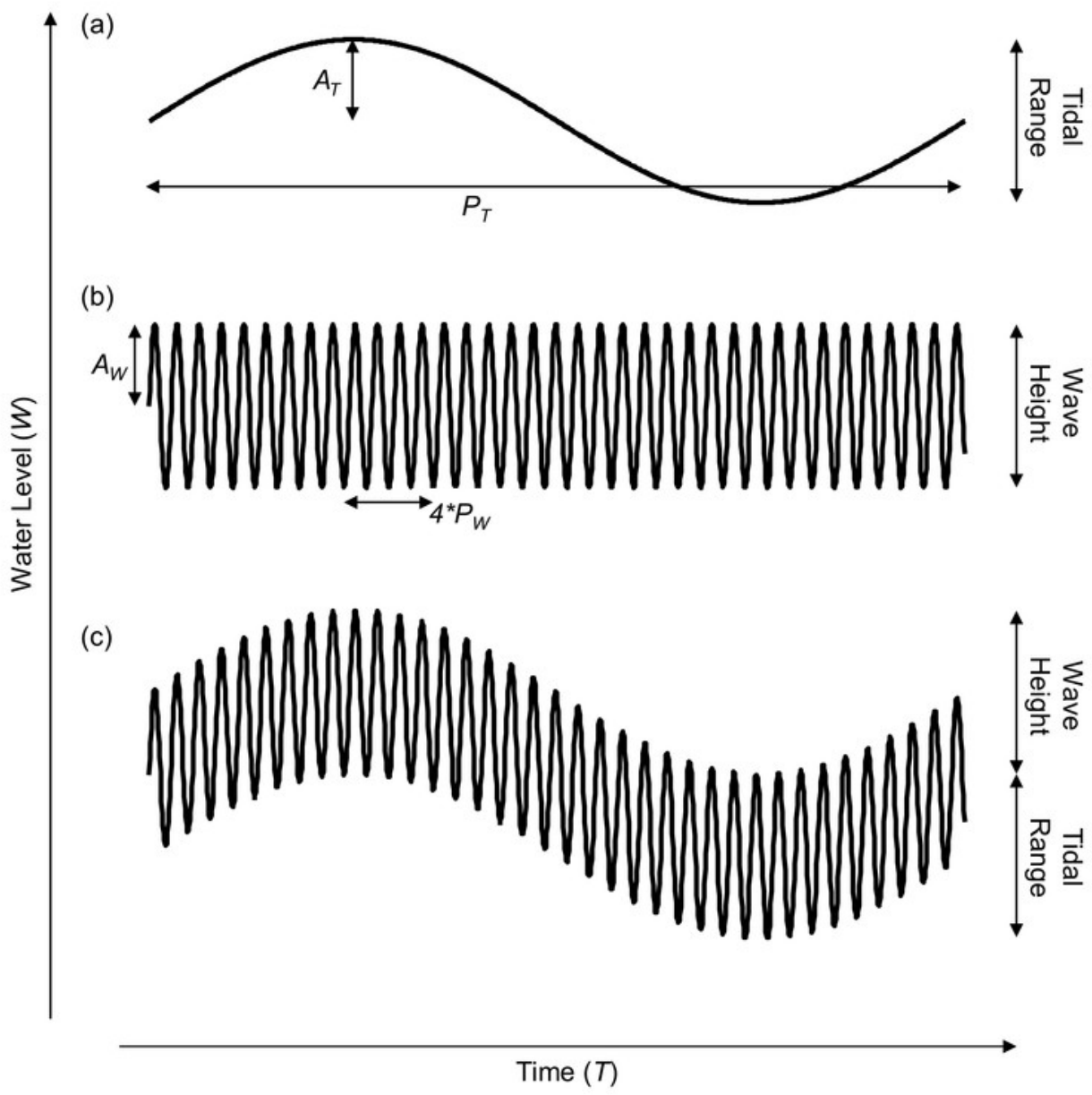




\section{Figure 2}

Wave-tide model of shoreline water level.

Using three ratios of wave height to tidal range: $0.1: 1(\mathrm{a}, \mathrm{c}, \mathrm{g}), 1: 1(\mathrm{~b}, \mathrm{e}, \mathrm{h})$, and 2:1 (c, $\mathrm{f}, \mathrm{i})$, we show shoreline water level and shoreline benchmarks. The four shoreline benchmarks predicted by the model (B1-B4) are demarcated by colored lines and the zones they bracket are shown in panels d-f. Relative wave energy, continuous emersion time and submersion time are diagramed in the conceptual models in panels $g-i$.

Fig 2

Tide-Dominated

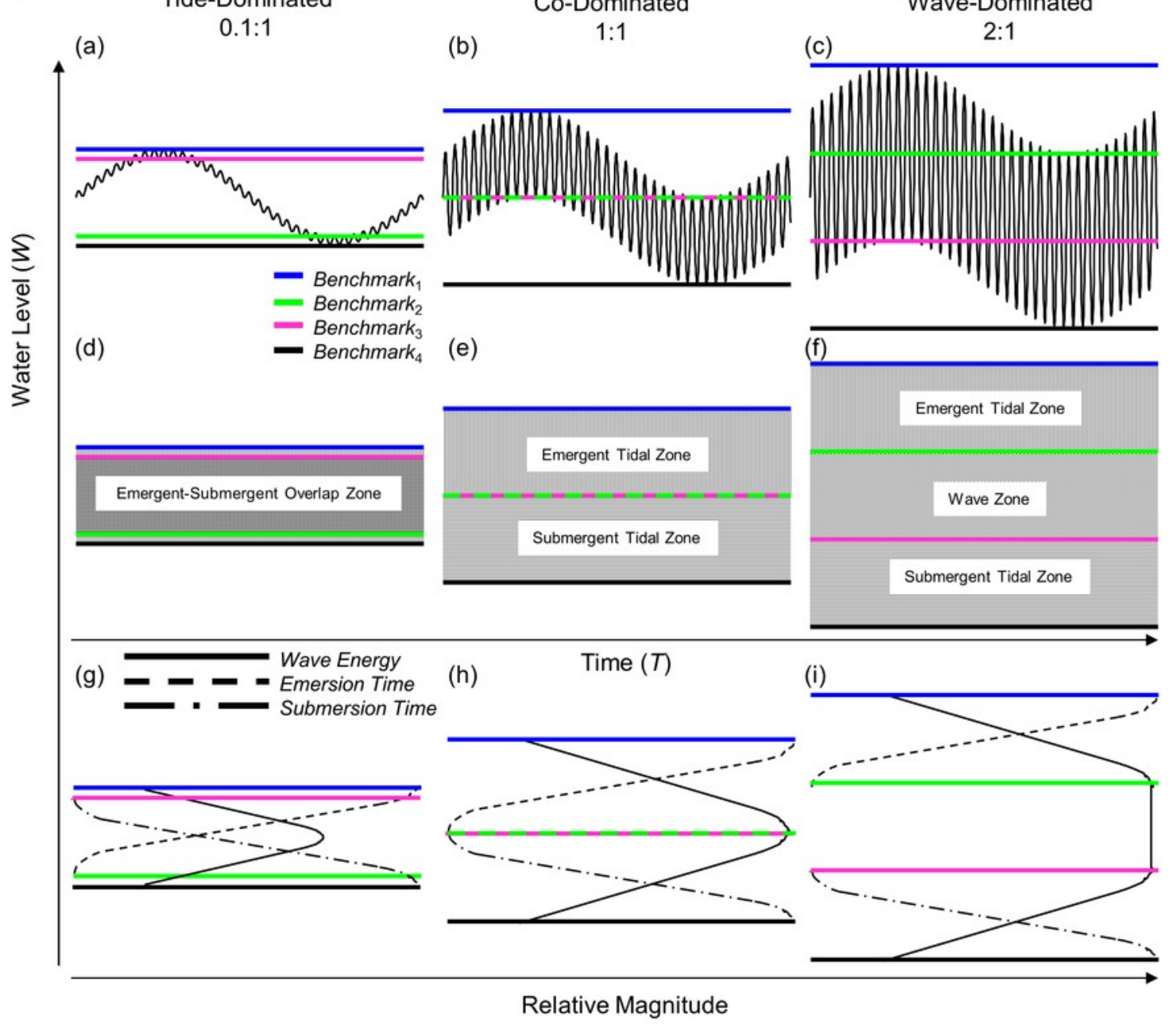




\section{Figure 3}

Global map classifying shorelines according to relative wave and tidal ranges.

(a) Shorelines classified as tide-dominated (mean diurnal tidal range $>>$ mean significant wave height), co-dominated (mean diurnal tidal range $\approx$ mean significant wave height), and wave-dominated (mean significant wave height $>>$ mean diurnal tidal range. (b) Ocean area color-coded by the ratio of wave height to tidal range. The locations of the sites featured in this study are indicated with stars - from left to right, Mokapu, Hawaii; Humboldt, California; and Portland Maine. 

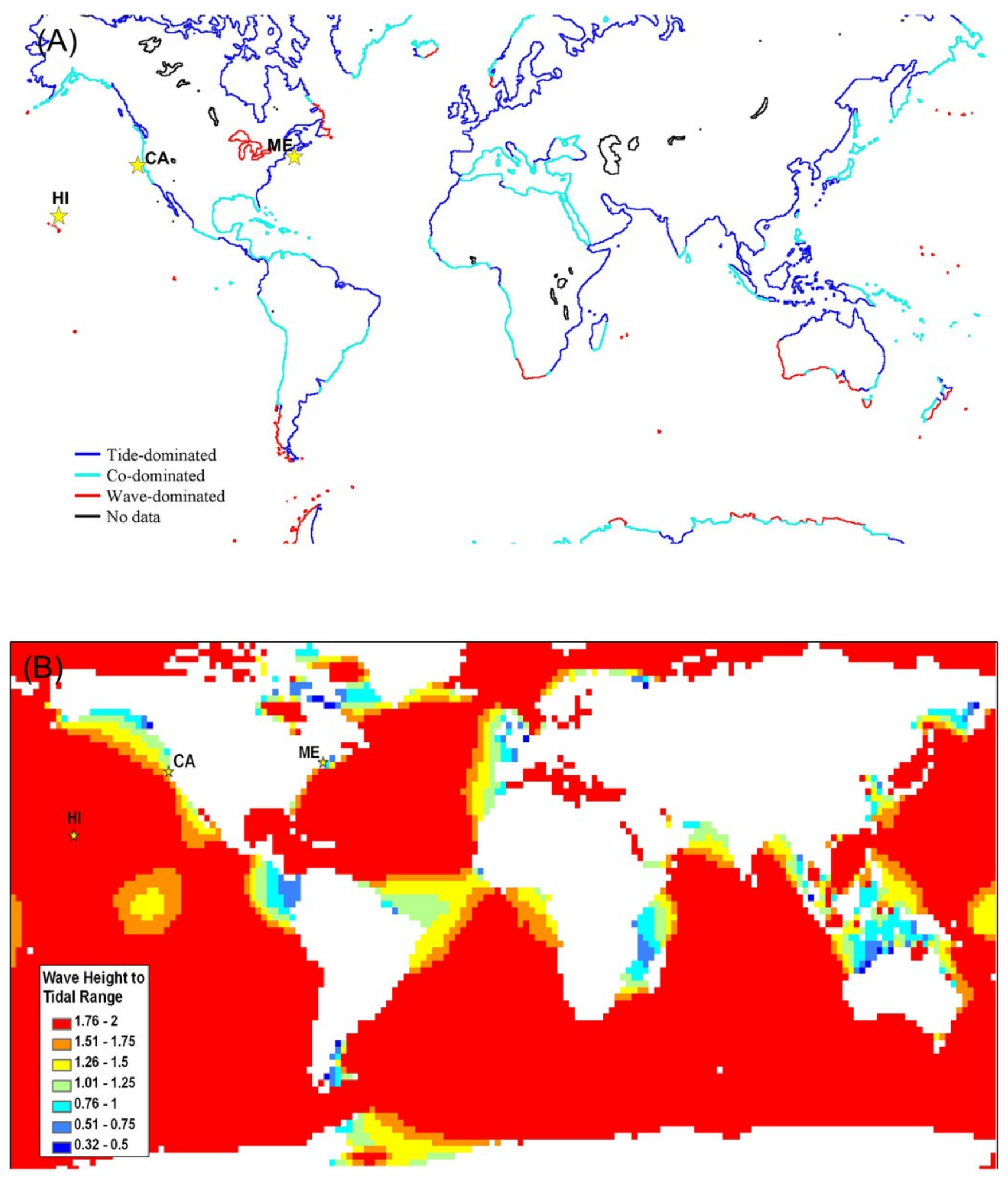

Fig 3 


\section{Figure 4}

Coastal water levels and physical zones across three representative sites.

Model of coastal water level (Equation 3, panels a-c), derived benchmarks and physical zones (Equations 4-7, panels $\mathrm{d}-\mathrm{i}$ ), and histograms of the hourly frequency of the ratio of wave height to tidal range (panels $j-1$ ) applied to three representative wave-exposed sites along a continuum from tide- to wave-domination (Portland, ME; Humboldt, CA; and Mokapu, HI respectively). Panels a-c display the water level during one tidal cycle (12.2 $\mathrm{h}$ oscillation period) from MHHW to MLLW at the yearly mean significant wave height (shorter oscillation period) plotted against time. Panels $d-f$ display the physical zones we derive from our model of coastal water level. Panels $\mathrm{g}$-i show the hourly determination of benchmark 1 ( blue line), benchmark 2 (green line), benchmark 3 (fuscia line), and benchmark 4 (black line), plotted against time from June 2005 to June 2006. Note that the rank positions of Benchmark 2 and Benchmark 3 switch from panels $\mathrm{a}, \mathrm{d}$, and $\mathrm{g}$ to panels $\mathrm{c}$, $\mathrm{f}$, and $\mathrm{i}$. 

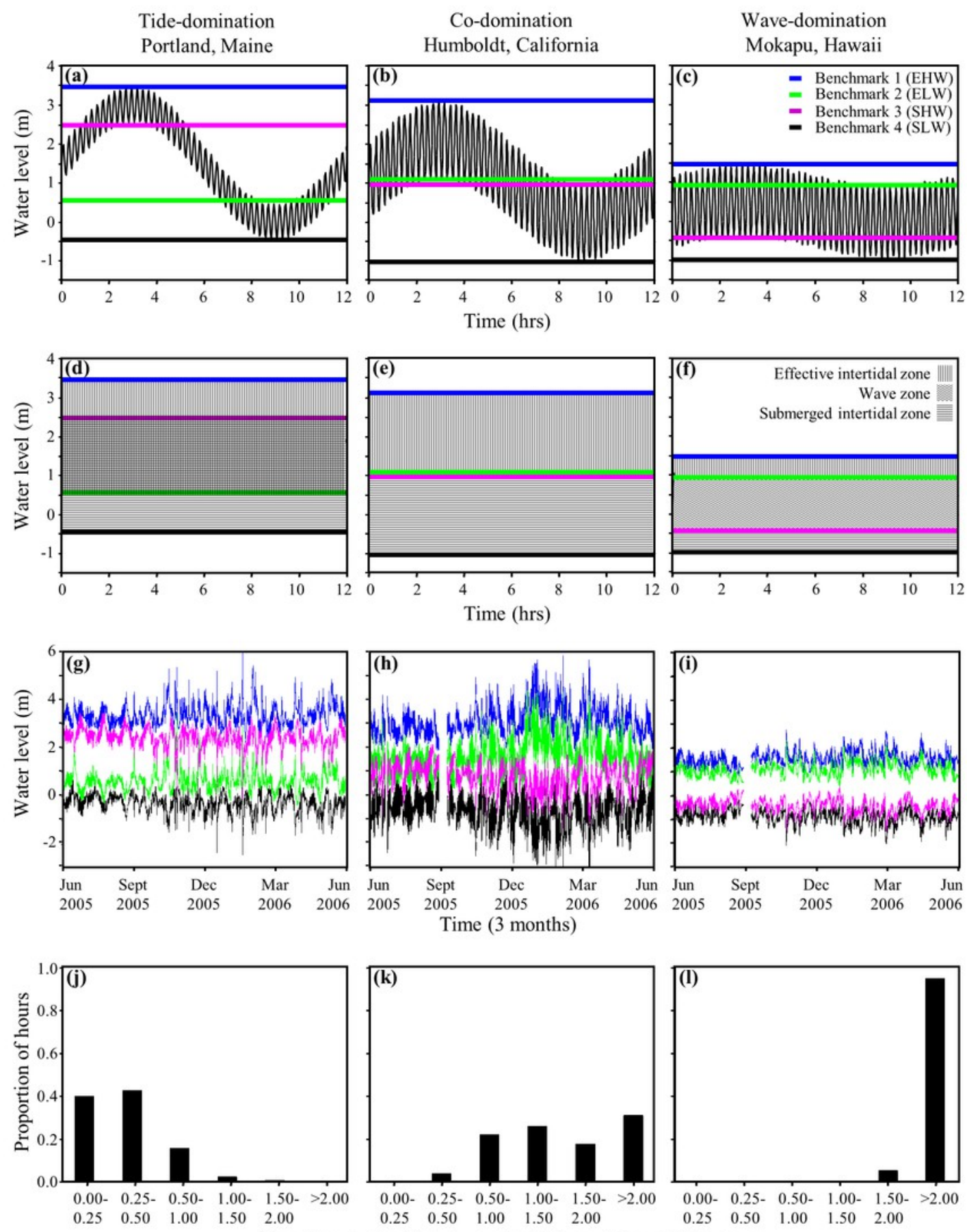

Mean hourly significant wave height : semidiurnal tidal range

Fig 4 


\section{Table 1 (on next page)}

Predominant physical conditions and their corresponding biological consequences for each of the zones predicted by the wave:tide zonation model.

Characterizations of predominant physical conditions and their biological consequences based on the assumption that the shores are equally wave-exposed. The predominant physical conditions and their corresponding biological consequences are ranked with zero to four "x" to indicate the magnitude of effect for each category column. 


\begin{tabular}{|c|c|c|c|c|c|c|c|c|c|c|c|c|c|}
\hline \multirow[b]{2}{*}{ Habitat } & \multicolumn{3}{|c|}{ Wave:Tide Regime } & \multicolumn{5}{|c|}{ Physical conditions } & \multicolumn{5}{|c|}{ Biological consequences } \\
\hline & $\begin{array}{l}\text { Tide } \\
\text { Dominated }\end{array}$ & $\begin{array}{l}\text { Co-domin } \\
\text { ated }\end{array}$ & $\begin{array}{l}\text { Wave } \\
\text { dominated }\end{array}$ & $\begin{array}{c}\text { Tidal } \\
\text { Emersion }\end{array}$ & $\begin{array}{c}\text { Tidal } \\
\text { Submersion }\end{array}$ & $\begin{array}{l}\text { Wave } \\
\text { wash }\end{array}$ & $\begin{array}{l}\text { Wave } \\
\text { force }\end{array}$ & $\begin{array}{c}\text { Relative } \\
\text { wave } \\
\text { energy }\end{array}$ & $\begin{array}{c}\text { Physiologica } \\
\text { l stress }\end{array}$ & $\begin{array}{l}\text { Physica } \\
\text { l stress }\end{array}$ & $\begin{array}{c}\text { Benthic } \\
\text { Predation } \\
\text { efficiency } \\
\end{array}$ & $\begin{array}{c}\text { Pelagic } \\
\text { Predation } \\
\text { efficiency } \\
\end{array}$ & $\begin{array}{l}\text { Terrestrial } \\
\text { Predation } \\
\text { efficiency } \\
\end{array}$ \\
\hline $\begin{array}{l}\text { Emergent } \\
\text { tidal zone }\end{array}$ & & Present & Present & $\mathrm{XXXX}$ & & $\mathrm{XX}$ & $\mathrm{XXXX}$ & $\mathrm{XX}$ & $\mathrm{XXXX}$ & $\mathrm{XX}$ & $\mathrm{XX}$ & & $\mathrm{XXXX}$ \\
\hline Wave zone & & 0 & Present & & & $\mathrm{XXXX}$ & XXXX & $\mathrm{XXXX}$ & & XXXX & $\mathrm{X}$ & $X$ & $\mathrm{X}$ \\
\hline $\begin{array}{r}\text { Submergent } \\
\text { tidal zone }\end{array}$ & & Prèsent & Present & & $\mathrm{XXXX}$ & $\mathrm{XX}$ & $\mathrm{XXXX}$ & $\mathrm{XX}$ & & $\mathrm{XX}$ & $\mathrm{XXXX}$ & $\mathrm{XXXX}$ & \\
\hline $\begin{array}{r}\text { Emergent-s } \\
\text { ubmergent } \\
\text { overlap } \\
\text { zone } \\
\end{array}$ & Present & $\sum_{\vec{\sigma}}^{\vec{c}}$ & & $\mathrm{XXXX}$ & $\mathrm{XXXX}$ & $\mathrm{X}$ & $\mathrm{XXXX}$ & $\mathrm{X}$ & XXXX & $\mathrm{X}$ & XXXX & $\mathrm{XXXX}$ & $\mathrm{XXXX}$ \\
\hline
\end{tabular}

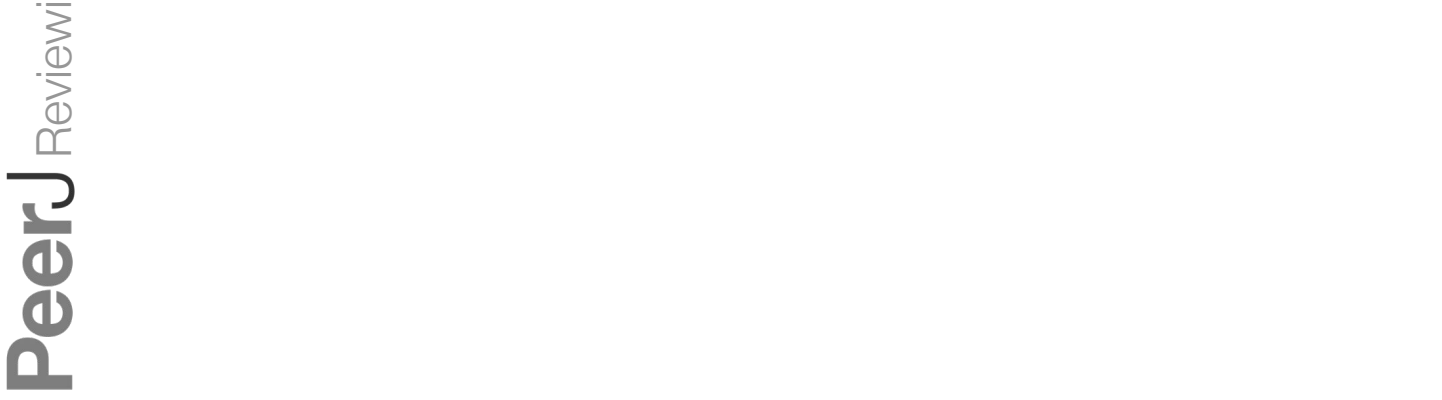

